\title{
Brief report: Compensatory health beliefs are negatively associated with intentions for regular fruit and vegetable consumption when self-efficacy is low
}

Journal of Health Psychology 2017, Vol. 22(8) 1094-1100 (C) The Author(s) 2016 Reprints and permissions: sagepub.co.uk/journalsPermissions.nav DOI: $10.1177 / 1359105315625358$ journals.sagepub.com/home/hpq ๑SAGE

\author{
Vera Storm', Dominique Reinwand ',2, \\ Julian Wienert ${ }^{3}$, Tim Kuhlmann ${ }^{4}$, Hein De Vries ${ }^{2}$ \\ and Sonia Lippke'
}

\begin{abstract}
Compensatory health beliefs (the beliefs that an unhealthy behaviour can be compensated by a healthy behaviour) can interfere with adherence to fruit and vegetable consumption recommendations. Fruit and vegetable consumption, social cognitive variables and compensatory health beliefs were investigated via self-report at baseline (T0) and 8-week follow-up (TI) in N=790 participants. Self-efficacy predicted fruit and vegetable consumption intentions. Planning mediated between intentions and TI fruit and vegetable consumption. Compensatory health beliefs negatively predicted intentions at low self-efficacy levels only. The results propose the use of self-efficacy interventions to diminish the negative effects of compensatory health beliefs when forming fruit and vegetable consumption intentions and foster planning to translate intentions into behaviour.
\end{abstract}

\section{Keywords}

cardiovascular disease, compensatory health beliefs, fruit and vegetable consumption, intention, self-efficacy

Coronary vascular diseases (CVD) are the main cause of mortality and morbidity in men aged over 45 years and women aged over 65 years in most developed European countries, including Germany and the Netherlands (Nichols et al., 2014). The prevalence of many of these conditions increases with age and is often affected by lifestyle factors such as physical inactivity and having an unhealthy diet (Mendis et al., 2011). There is accumulating evidence that especially fruit and vegetable consumption (FVC) plays an important role in preventing other chronic conditions associated with CVD, such as diabetes mellitus or (future) cardiovascular issues
(Boeing et al., 2012). Despite the widely used recommendations to eat at least five portions of fruit and vegetable a day, few adults adhere to these guidelines (Organisation for Economy, Cooperation and Development (OECD), 2013).

\footnotetext{
IJacobs University Bremen, Germany

2Maastricht University, Netherlands

3University of Lübeck, Germany

${ }^{4}$ University of Konstanz, Germany

Corresponding author:

Vera Storm, Jacobs University Bremen, Campus Ring

I, Bremen 28759, Germany.

Email: v.storm@jacobs-university.de
} 
Models and theories in health psychology are usually founded upon the common 'metatheory' that some form of intention is central to initiating health behaviour or behaviour change. However, compensatory health beliefs (CHBs; Rabiau et al., 2006) can interfere with successful health behaviour change. CHBs implicate the belief that the negative consequences of an unhealthy behaviour (e.g. eating unhealthily) can be compensated by engaging in another, more healthy behaviour (e.g. exercising). In the Compensatory Carry-Over Action Model (CCAM; Lippke, 2014), CHBs are proposed to be disturbing in the motivational phase of behaviour change and they can be harmful if negatively related to behavioural intentions. Negative effects of CHBs on intentions have already been found in the context of physical activity (Berli et al., 2013) and dietary intake (Radtke et al., 2014). These negative effects imply that high CHBs are associated with low intentions to show the target behaviour. Nonetheless, the effects of CHBs on intention may also be influenced by certain motivational variables. However, moderation effects have been widely overlooked, with the exception of one study by Radtke et al. (2014), finding that CHBs were positively related to physical activity intentions in women with high levels of risk perception. This could imply that when people perceive high risks and observe possibilities to compensate low physical activity levels with other behaviours, they still have positive intentions, regardless of high CHBs.

In accordance with Rabiau et al. (2006), it is likely that the effect of CHBs on intentions also depends on self-efficacy beliefs, whereby individuals with low levels of self-efficacy are less likely to behave according to their intentions (Ochsner et al., 2013). Therefore, individuals low in self-efficacy might be more likely to subdue their temptations and show CHBs, which negatively influence intentions to undertake a health behaviour. Even if people have developed intentions to perform such a behaviour, they fail to translate them into action, which is commonly referred to as the intentionbehaviour gap (Webb and Sheeran, 2006).
Intervention research tries to bridge this gap with self-regulation strategies such as planning (Hagger and Luszczynska, 2014). Through specifying when and where a certain behaviour will be carried out or how potential barriers will be tackled, the goal behaviour is more likely to be shown. Indeed, this has already been found in the field of FVC (Adriaanse et al., 2011), especially when self-efficacy beliefs are high (Luszczynska and Haynes, 2009). However, research including intentions, CHBs and planning has been scarce to date.

Given the little research on CHBs thus far, the direction of the effect of CHBs on intentions and behaviour needs to be tested more systematically. Accordingly, this longitudinal study aims to examine the role of CHBs in predicting FVC intentions and actual FVC among people motivated to reduce their cardiovascular risk in a longitudinal randomised controlled trial (RCT). First, in accordance with Radtke et al. (2014) and Lippke (2014), we assume that CHBs negatively interrelate with FVC intentions (hypothesis 1). Furthermore, as Rabiau et al. (2006) posit, people with low levels of self-efficacy lack the necessary self-control to realise health goals, which means that they are more likely to have $\mathrm{CHBs}$ and less likely to show intentions for regular FVC. Therefore, we hypothesise that the negative effect of CHBs on intentions is augmented by low levels of selfefficacy (hypothesis 2). Finally, we hypothesise that planning mediates between intentions and follow-up FVC (hypothesis 3), as already shown for various health behaviours besides FVC (De Vries et al., 2014; Hagger and Luszczynska, 2014; Reuter et al., 2010).

\section{Method}

\section{Participants and procedure}

In all, 790 study participants were recruited by the authors in cardiac rehabilitation facilities, heart training groups, online panels and different Internet platforms in Germany and the Netherlands. Participation in the study was voluntary and data were anonymised. No 
incentives for participation were provided. The inclusion criteria were as follows: being at least 20 years old, no contra-indications for physical activity and $\mathrm{FVC}$, having an interest in reducing cardiovascular risk, sufficient reading and writing skills in the relevant language, and Internet access and Internet literacy. The follow-up (T1) with $n=209$ took place 9 weeks after the T0 baseline measurement. This study was designed as a RCT to investigate whether a computer-tailored intervention is effective in increasing self-reported physical activity and FVC. For the present analyses, the quasi-experimental longitudinal design does not hold interest. The T0 and T1 questionnaires were the same for the intervention and control group. $T$-tests revealed no baseline differences between the intervention and control group. Specific information on the study design has been published elsewhere (Reinwand et al., 2013).

\section{Measures}

Besides socio-demographic information (i.e. gender, year of birth), height (in $\mathrm{cm}$ ) and body weight (in $\mathrm{kg}$ ) were assessed to calculate body mass index (BMI). All mentioned measures were obtained via web-based selfreport questionnaires.

CHBs. The CHB scale by Knäuper et al. (2004) proved valid regarding construct- and criterionrelated validity. From the original $\mathrm{CHB}$ scale, items related to dieting were used and slightly revised in this study. Therefore, the scale comprised only four items (Cronbach's $\alpha=.72$ ); for example, 'If one is physically active, it's okay to eat as much as one wants to'. Participants rated all answers of compensatory cognitions from do not agree at all (1) to agree completely (7) (Lippke et al., 2015).

Study participants indicated the following social cognitive items on Likert scales ranging from 'not true (1)' to 'exactly true (7)'. All social cognitive measures had been validated in previous studies (Lippke et al., 2009; Luszczynska et al., 2007).
FVC intentions. Intentions concerning FVC were assessed using the item: 'I seriously intent to eat at least five portions of fruit and vegetable daily'.

Self-efficacy. Self-efficacy towards eating fruit and vegetable (Cronbach's $\alpha=.92$ ) was assessed with five items; for example, 'I am certain that I can eat five portions of fruit and vegetable a day even it is difficult'.

Planning. Planning was assessed with the use of six items (Cronbach's $\alpha=.92$ ), such as, 'For the next month I already planned in detail at which meals I will eat fruits and vegetables (e.g. additional salad)'.

FVC. FVC on a typical day during the last 7 days was assessed using four items, whereby study participants reported their (1) daily consumption of portions of fruit, (2) cooked or steamed vegetables, (3) salad and raw vegetables, and (4) glasses of fruit and vegetable juice. Portions were added to a daily FVC sum score.

\section{Statistical analysis}

Data analysis was conducted with SPSS 22 and SPSS 22 AMOS, using structural equation modelling to explore the links between the mentioned constructs and validate the full model. Missing values $(0 \%-19 \%)$ were estimated with full information maximum likelihood (FIML) methods. For moderation analysis (hypothesis 2), the independent variables selfefficacy and CHBs were $z$-standardised and multiplied for the interaction term. For the simple slopes analysis, we grouped self-efficacy into three categories (low, medium, high). Mediation analysis with bootstrapping (5.000 samples) according to Preacher and Hayes (2004) was undertaken to test hypothesis 3. For the analyses, gender, country of residence, age, BMI, marital status, employment status, group condition (intervention group vs. waiting control group), baseline FVC and baseline planning were included as covariates. Results of indicate BMI differences regarding the relationship 
Table I. Summary of the main study variables and baseline equivalency.

\begin{tabular}{|c|c|c|c|c|c|}
\hline & Total $(N=790)$ & German $(n=37 I)$ & Dutch $(n=419)$ & & \\
\hline & $M(\mathrm{SD})$ & $M(\mathrm{SD})$ & $M(S D)$ & $T$-test & $p$ \\
\hline Age & $50.85(12.5 I)$ & $52.18(11.18)$ & $49.67(12.85)$ & 8.51 & .004 \\
\hline BMI & $27.55(5.4 I)$ & $28.02(5.56)$ & $27.13(5.24)$ & 5.38 & .021 \\
\hline TO FVC intention & $4.75(1.52)$ & $4.56(1.88)$ & $4.92(1.62)$ & 8.37 & .004 \\
\hline TO CHBs & $2.65(1.00)$ & $2.72(1.14)$ & $2.58(0.86)$ & 3.61 & .058 \\
\hline T0 FVC self-efficacy & $4.66(1.46)$ & $4.81(1.45)$ & $4.52(1.44)$ & 7.78 & .005 \\
\hline TI FVC planning & $4.06(2.17)$ & $3.92(1.73)$ & 4.17 (1.39) & 1.33 & .251 \\
\hline \multirow[t]{2}{*}{ TI FVC } & $4.09(2.17)$ & $3.04(1.28)$ & $4.89(2.37)$ & 44.61 & $<.001$ \\
\hline & $N(\%)$ & $n(\%)$ & $n(\%)$ & $\chi^{2}$ & $p$ \\
\hline Gender, female & 497 (62.9) & $213(57.4)$ & $284(67.8)$ & 9.07 & .004 \\
\hline
\end{tabular}

between fruit and vegetable intake (FVI) and the health action process approach (HAPA)based social cognitive variables.

\section{Results}

\section{Sample characteristics}

The sample had a mean age of 50.85 years $(S D=12.15$, range: $20-84), 62.9$ per cent were females $(n=497), 71.8$ per cent $(n=646)$ of the participants were married or in a relationship and 52.5 per cent $(n=415)$ were employed either fullor part-time. Table 1 provides an overview of all descriptive variables and baseline equivalency.

\section{Preliminary analysis and missing values}

There were no significant differences between those who participated in both measurement points and those who dropped out after T0 $(n=581)$ regarding gender $\left(\chi^{2}(1, N=789)=2.08\right.$, $p=.149), \quad$ country $\quad \chi^{2}(1, \quad N=789)=1.20$, $p=.274)$, age $(F(1,789)=1.11, p=.293)$, BMI $(F(1,789)=0.38, p=.535)$, baseline CHBs $(F(1,789)=0.06, \quad p=.812)$, baseline $\mathrm{FVC}$ intentions $(F(1,789)=0.03, p=.776)$, baseline self-efficacy $(F(1,789)=0.63, p=.430)$, baseline planning $(F(1,789)=0.66, p=.417)$ and baseline $\mathrm{FVC}(F(1,789)=2.49, p=.131)$. T0 T1 attrition was 73.9 per cent $(n=584)$.

\section{Specification of the conceptual model and testing of the hypotheses}

The full proposed model is portrayed in Figure 1 and shows an acceptable model fit $\chi^{2}(27$, $N=790)=5.20, p<.001$, comparative fit index $(\mathrm{CFI})=.91$, Tucker-Lewis index $(\mathrm{TLI})=.72$, root mean squared error of approximation $($ RMSEA $)=.07(.06 ; .08))$ to the data.

In contrast to hypothesis $1, \mathrm{CHBs}$ did not interrelate with FVC intentions directly $(\beta=-.05, p=.114)$. However, in line with hypothesis 2 , the interaction between CHBs and self-efficacy predicted FVC intentions ( $\beta=.07, p=.020)$, thus indicating a moderation effect of self-efficacy with CHBs, whereby CHBs negatively influenced FVC intentions for those with low self-efficacy only. The simple slopes analyses demonstrated that the higher the CHBs, the lower the intentions for FVC in participants with low levels of selfefficacy $(t(780)=-2.55, p=.011)$, but not those with medium $(t(780)=-1.32, p=.187)$ nor high levels $(t(780)=0.87, p=.384)$.

In line with hypothesis $3, \mathrm{~T} 1$ planning mediated between $\mathrm{T} 0 \mathrm{FVC}$ intentions and $\mathrm{T} 1 \mathrm{FVC}$ : the effect of T0 FVC intention on T1 FVC was not significant $(\beta=.09, p=.215)$. However, the effect of T0 FVC intentions on T1 planning was $\beta=.14(p=.031)$ and the effect of T1 planning on T1 FVC was $\beta=.16(p=.022)$. The bootstrapped standardised indirect effect of $\mathrm{T} 0 \mathrm{FVC}$ 


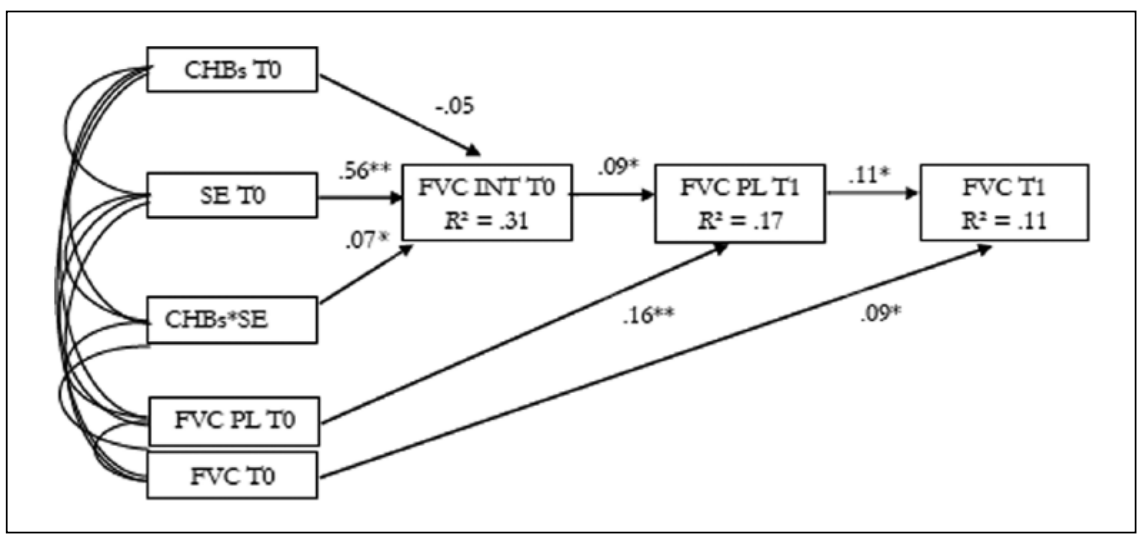

Figure I. Conceptual model with standardised regression coefficients showing the longitudinal effect of $\mathrm{CHBs}$ in the health behaviour change process for fruit and vegetable consumption controlling for age, marital status, employment status, gender, group, country of residence, and BMI.

Note: $\mathrm{TO}=$ baseline; $\mathrm{TI}=$ follow-up at 8 weeks; $\mathrm{CHBs}=$ compensatory health beliefs; $\mathrm{SE}=$ self-efficacy, $\mathrm{FVC}=$ fruit and vegetable consumption; $\mathrm{PL}=$ planning; $\mathrm{INT}=$ intention; $N=790$.

$* p<.05, * * p<.001$.

intentions on T1 FVC via T1 planning was $\beta=.03$ (95\% confidence interval (CI): .01-.15).

Self-efficacy significantly interrelates with intentions $(\beta=.56, p<.001)$, indicating that high self-efficacy levels are associated with high intentions. In addition, baseline FVC significantly predicted follow-up behaviour FVC $(\beta=.09, p=.002)$. The explained variances are portrayed in Figure 1.

\section{Discussion}

This longitudinal study aimed at examining the role of CHBs in predicting FVC intentions and actual $\mathrm{FVC}$ among people motivated to reduce their cardiovascular risk. CHBs within the health behaviour change process for FVC held special interest in this study. In contrast to Radtke et al. (2014), we found that CHBs did not directly interrelate with intentions to eat fruit and vegetables. Nonetheless, CHBs are negatively interrelated with intentions when self-efficacy levels were particularly low. One possible explanation might be that when selfefficacy exceeds a certain threshold, CHB cannot exhibit its conflicting effect on intentions. This is in line with Rabiau et al. (2006), given that individuals high in self-efficacy are more likely to resist temptations and practice barrier relapse management. Higher levels of self-efficacy were associated with higher intentions to eat fruit and vegetables, which is a typical finding in health behaviour research (Luszczynska et al., 2007; Luszczynska and Haynes, 2009). This suggests that improving a person's confidence in their ability (e.g. by mastery experience) to eat in a healthy way may set the stage for successful behaviour change by strengthening intentions and diminishing the negative effects of CHBs, for instance.

Higher intentions lead to more planning, which subsequently enables higher levels of FVC at a later stage. This is in accordance with previous findings on the effects of planning (Hagger and Luszczynska, 2014; Reuter et al., 2010) and suggests the further use of self-regulatory strategies in supporting people to change to healthier lifestyles.

\section{Limitations and future research}

This study is not without limitations; first, the high dropout needs to be addressed. Although there were no differences between dropouts and those who completed both waves of measurements, 
future studies should aim to obtain larger sample sizes, especially at follow-up.

Second, the dietary behaviour data here are based upon self-report only and might be prone to bias, such as recall bias. Future studies should consider more objective measures in addition to self-report such as biomarkers, which are already frequently applied in dietary research (Combs et al., 2013).

A third limitation refers to the measurement of subjects' CHBs, which were measured with four items for parsimonious reasons only. Despite showing good internal consistency among our sample, future researchers might want to measure $\mathrm{CHBs}$ with more sophisticated fruit- and vegetable-specific scales and investigate whether and how compensatory behaviour is actually carried out as 'believed' earlier.

Finally, there were small yet significant differences between German and Dutch participants regarding the measures that we used. Nonetheless, we assume that such differences do not relate to the respective countries, as both countries have similar nutrition recommendations (WHO, 2003, 2006) and prevention campaigns (e.g. ' 5 a day'; Deutsche Gesellschaft für Ernährung e.V., 2012; Voedingscentrum Den Haag, 2011).

\section{Conclusion}

This study provides new insights into the concept of CHBs, with our results highlighting the negative effect of CHBs in the intention formation process for regular FVC, particularly at low levels of self-efficacy. Implications for theory development and practice could be as follows: future researchers might want to include extra self-efficacy modules (e.g. selfefficacy boosting exercises) to ensure diminishing the negative effect of CHBs when forming intentions to eat healthy or tailor an intervention to self-efficacy levels of participants, whereby people low in self-efficacy can profit equally in terms of intention formation. Moreover, planning components should be integrated to help translate people's good intentions into action. Especially people who are at risk of developing cardiovascular diseases should be made aware of the tendency to form CHBs (i.e. through discussions with medical professionals or eHealth programmes) and how to couple these maladaptive thoughts with goal-oriented planning strategies to stick to their health goals.

\section{Declaration of Conflicting Interests}

The author(s) declared no potential conflicts of interest with respect to the research, authorship and/or publication of this article.

\section{Funding}

The author(s) disclosed receipt of the following financial support for the research, authorship, and/or publication of this article: This research was funded by the Wilhelm-Stiftung für Rehabilitationsforschung within the Donors' Association for the Promotion of Humanities and Sciences in Germany.

\section{References}

Adriaanse MA, Vinkers CDW, Hox JJ, et al. (2011) Do implementation intentions help to eat a healthy diet? A systematic review and metaanalysis of the empirical evidence. Appetite 55: 181-193.

Berli C, Loretini P, Radtke T, et al. (2013) Predicting physical activity in adolescents: The role of compensatory health beliefs within the health action process approach. Psychology \& Health 29(4): 458-474.

Boeing H, Bechthold A, Bub A, et al (2012) Critical review: Vegetables and fruit in the prevention of chronic diseases. European Journal of Nutrition 51(6): 637-663.

Combs GF Jr, Trumbo PR, McKinley MC, et al. (2013) Biomarkers in nutrition: New frontiers in research and application. Annals of the New York Academy of Sciences 1278: 1-10.

Deutsche Gesellschaft für Ernährung e.V. (2012) Gemüse und Obst in der Prävention ausgewählter chronischer Krankheiten. Available at: https:/www.dge.de/fileadmin/public/doc/ws/ stellungnahme/DGE-Stellungnahme-GemueseObst-2012.pdf (accessed 7 December 2015).

De Vries H, Eggers SM, Lechner L, et al. (2014) Predicting fruit consumption: The role of habits, previous behaviour and mediation effects. $B M C$ Public Health 14: 730. 
Hagger MS and Luszczynska A (2014) Implementation intention and action planning interventions in health contexts: State of the research and proposals for the way forward. Applied Psychology: Health and Well-being 6(1): 1-47.

Knäuper B, Rabiau M, Cohen O, et al. (2004) Compensatory health beliefs scale development and psychometric properties. Psychology and Health 19(5): 607-624.

Lippke S, Hohmann C, Kalusche A, et al. (2015) Compensatory beliefs scale-general and diet (German). Available at: http://www.psych. mcgill.ca/perpg/fac/knaeuper/GermanCHB.doc (accessed 7 December 2015).

Lippke S, Ziegelmann JP, Schwarzer R, et al. (2009) Validity of stage assessment in the adoption and maintenance of physical activity and fruit and vegetable consumption. Health Psychology 28(2): 183-193.

Lippke S (2014) Modelling and supporting complex behavior change related to obesity and diabetes prevention and management with the compensatory carry-over action model. Journal of Diabetes and Obesity 1(1): 1-5.

Luszczynska A, Tryburcy M and Schwarzer R (2007) Improving fruit and vegetable consumption: A self-efficacy intervention compared with a combined self-efficacy and planning intervention. Health Education Research 22(5): 630-638.

Luszczynska A and Haynes C (2009) Changing nutrition, physical activity and body weight among student nurses and midwives. Effects of a planning intervention and self-efficacy beliefs. Journal of Health Psychology 14(8): 1075-1084.

Mendis S, Puska P and Norvving B (2011) Global atlas on cardiovascular disease prevention and control. Available at: http://www.who.int/ cardiovascular_diseases/resources/atlas/en/ (accessed 07 December 2015).

Nichols M, Townsend N, Scarborough P, et al. (2014) Cardiovascular disease in Europe 2014: Epidemiological update. European Heart Journal 35(42): 2950-2959.

Ochsner S, Scholz U and Hornung R (2013) Testing phase-specific self-efficacy beliefs in the context of dietary behaviour change. Applied Psychology: Health and Well-being 5(1): 99-117.
Organisation for Economy, Cooperation and Development (OECD) (2013) Health at a Glance: OECD Indicators. Paris: OECD Publishing.

Preacher KJ and Hayes A (2004) SPSS and SAS procedures for estimating indirect effects in simple mediation models. Behaviour Research Methods, Instruments, \& Computers 36: 717-731.

Rabiau M, Knäuper B and Miquelon P (2006) The eternal quest for optimal balance between maximising pleasure and minimising harm: The compensatory health beliefs model. British Journal of Health Psychology 11(1): 139-153.

Radtke T, Kaklamanou D, Scholz U, et al. (2014) Are diet-specific compensatory health beliefs predictive of dieting intentions and behaviour? Appetite 76: 36-43.

Reinwand D, Kuhlmann T, Wienert J, et al. (2013) Designing a theory- and evidence-based tailored eHealth rehabilitation aftercare program in Germany and the Netherlands: Study protocol. BMC Public Health 13: 1081.

Reuter T, Ziegelmann JP, Wiedemann AU, et al. (2010) Changes in intentions, planning, and self-efficacy predict changes in behaviors. An application of latent true change modeling. Journal of Health Psychology 15(6): 935-947.

Voedingscentrum Den Haag (2011) Gezonde basisvoeding met de schijf van vijf factsheet. Available at: http://www.voedingscentrum. nl/Assets/Uploads/Documents/Factsheet $\% 20$ Gezonde $\% 20$ basisvoeding $\% 20$ met $\% 20$ de\%20Schijf\%20van\%20Vijf.pdf (accessed 7 December 2015).

Webb TL and Sheeran P (2006) Does changing behavioural intentions engender behaviour change? A meta-analysis of the experimental evidence. Psychological Bulletin 132: 249-268.

World Health Organization (WHO) (2003) Food based dietary guidelines in the WHO European region. Available at: http://www.euro.who. int/_data/assets/pdf_file/0017/150083/ E79832.pdf (accessed 7 December 2015).

World Health Organization (WHO) (2006) Comparative analysis of nutrition policies in the WHO European region (no. EUR/06/5062700/ BD/2). Available at: http://www.euro.who.int/ data/assets/pdf_file/0004/149782/instanbul_ conf_20ebd02.pdf (accessed 7 December 2015). 